\title{
The Prevalence of Growth Variations Among Pediatric Celiac Disease Patients at the Time of Diagnosis
}

Ghassan Sukkar ${ }^{1}$, Alhusain M. Alshareef ${ }^{2}$, Marwan Aljahani ${ }^{2}$, Hadeel A. Alharthi ${ }^{3}$,Abdulaziz Fakieha

1. Pediatrics, King Saud Bin Abdulaziz University for Health Sciences College of Medicine, Jeddah, SAU 2. Medicine, King Abdulaziz Medical City/King Saud bin Abdulaziz University for Health Sciences, Jeddah, SAU 3. Faculty of Medicine, Ibn Sina National College, Jeddah, SAU

Corresponding author: Ghassan Sukkar, sukkar003@gmail.com

\section{Abstract}

\section{Background and aim}

Celiac disease is an immune-mediated disorder caused by sensitivity to dietary gluten. Celiac patients typically present with malabsorption and low growth parameters; however, studies have shown that the presentation of celiac disease can have a higher percentage of patients with normal or high growth parameters and no signs of malabsorption. The study aims to estimate the prevalence of the growth variation found in children with biopsy-confirmed celiac disease at the time of diagnosis.

\section{Methods}

We included 31 biopsy-confirmed pediatric celiac patients diagnosed from 2007 to 2018 in King Abdulaziz Medical City, Jeddah, Saudi Arabia. Patients' height, weight, and BMI at the time of diagnosis were converted to z-scores and growth percentiles according to the Centers for Disease Control and Prevention growth charts. In addition, patients' comorbid conditions were also recorded.

\section{Results}

At the time of diagnosis, $45.16 \%$ of our patients presented as underweight, $41.94 \%$ of patients had normal weight, $6.5 \%$ were overweight and obese, respectively. The mean BMI was $15.44( \pm 3.65)$. Our population had a statistically significant lower BMI, height, and weight mean z-scores at the time of diagnosis.

\section{Conclusion}

A significant number of children diagnosed with celiac disease in our center had low weight, height, and BMI at the time of diagnosis. However, we emphasize that having normal growth parameters does not rule out the diagnosis of celiac disease.

Review began 10/28/2020 Review ended 11/16/2020 Published 11/25/2020

() Copyright 2020 Sukkar et al. This is an open access article distributed under the terms of the Creative Commons Attribution License CC-BY 4.0., which permits unrestricted use, distribution, and reproduction in any medium, provided the original author and source are credited.
Categories: Pediatrics, Gastroenterology

Keywords: tissue transglutamase, celiac disease

\section{Introduction}

Celiac disease (CD) is a chronic immune-mediated inflammatory disorder of the small bowel caused by sensitivity to dietary gluten as well as other related prolamins in individuals who are genetically susceptible [1]. The prevalence of celiac disease is estimated to affect 0.5 to one percent of the general population in many countries. In the United States, Australia, and Europe, the prevalence is estimated to range from 1:80 to 1:300 children [2]. Locally, according to a meta-analysis conducted by Safi MA, CD prevalence in Saudi Arabia is 1.4 percent [3]. This condition is characterized by both intestinal and extraintestinal manifestations, including diarrhea, dermatitis herpetiformis, anemia, short stature, and poor weight gain [4, 5]. The diagnosis of celiac disease is supported by positive serological tests such as tissue transglutaminase antibodies (tTG) and anti-endomysial antibodies (EMA). However, a small intestinal mucosal biopsy remains the gold standard for establishing a diagnosis [6]. The only effective treatment currently available for celiac disease is a strict life-long gluten-free diet (GFD) [2, 7]. The typical form of celiac disease is characterized by failure to thrive, malnutrition, and weight loss, especially in young children [8, 9]. However, over time the presentation of celiac disease has changed. Despite the known typical presentation of the disease, many studies show that the condition may also be associated with normal weight or overweight [5]. In a cohort study conducted in the United States, the percentage of normal weight and overweight patients was significantly higher than underweight patients at the time of diagnosis [10]. This implies that malnutrition is not necessarily always present at the time of diagnosis. Our aim in this study is to estimate the growth variation found in children diagnosed with celiac disease via biopsy at the time of the diagnosis. 


\section{Materials And Methods}

We conducted a single-center cross-sectional chart review on pediatric celiac patients from 2007 to 2018 at King Abdul-Aziz Medical City (KAMC), Jeddah, Saudi Arabia. We included all patients aged from two to 14 years with biopsy-confirmed celiac disease diagnosis according to the Marsh criteria. Patients with genetic diseases that affect growth, and patients receiving growth-enhancing medications were excluded from the study. We used Raosoft software (Roasoft, Inc., US) to calculate our sample size. We used a margin of error of $5 \%$ and a confidence interval of $95 \%$. The recommended sample size was 296 . Patients' height, weight, and BMI were converted to age-specific percentiles and z-scores derived from growth charts published by the Centers for Disease Control and Prevention (CDC). Additional information regarding the patients' presentation, comorbidities, and serological markers were also collected. SAS software 9.4 (SAS Institute Inc., Cary, US) was used for statistical analysis. Categorical variables were described as frequencies and percentages, and continuous variables were described as mean and standard deviation. T-test was used to compare the means of the z-scores, Mann-Whitney test was used to compare two independent groups.

\section{Results}

Out of 66 patients, 31 patients fulfilled the research selection criteria (Figure 1). Their demographics and clinical characteristics are presented in Table 1. Nineteen (61.29\%) were males, and 12 (38.71\%) were females. The mean age was $7.71( \pm 3.09)$. The mean weight, height, and BMI were: $23.45( \pm 12.18), 119.77$ $( \pm 18.27), 15.44( \pm 3.65)$, respectively. Their corresponding z-scores were: $-1.37( \pm 1.80),-0.92( \pm 1.46)$ and 1.28 ( \pm 1.93$)$. Fourteen $(45.16 \%)$ patients were underweight, 13 (41.94\%) were normal weight, two (6.45\%) were overweight, and two (6.45\%) were obese (see Figure 2). In terms of height, 18 (58.06\%) had normal stature, 11 (35.48\%) were short stature, and two (6.45\%) were tall stature (see Figure 3). Nineteen (61.29\%) patients were associated with different comorbidities in the following frequencies: diabetes mellitus type 1 (DMI) - nine (29.03\%), asthma - five (16.12\%), anemia - two (6.45\%), attention deficit hyperactivity disorder (ADHD) - two (6.45\%), and autism - one (3.22\%). The mean anthropometric measures of CD patients were significantly different from the norm (z-score of zero) with p values of 0.0002 for mean weight, 0.0016 for mean height, and 0.0004 for mean BMI (Table 2). On the other hand, no statistical differences in these measurements were found when we compared comorbid patients with non-comorbid ones since the p-values were 0.62 for mean weight, 0.73 for mean height, and 0.73 for mean BMI (Table 3).

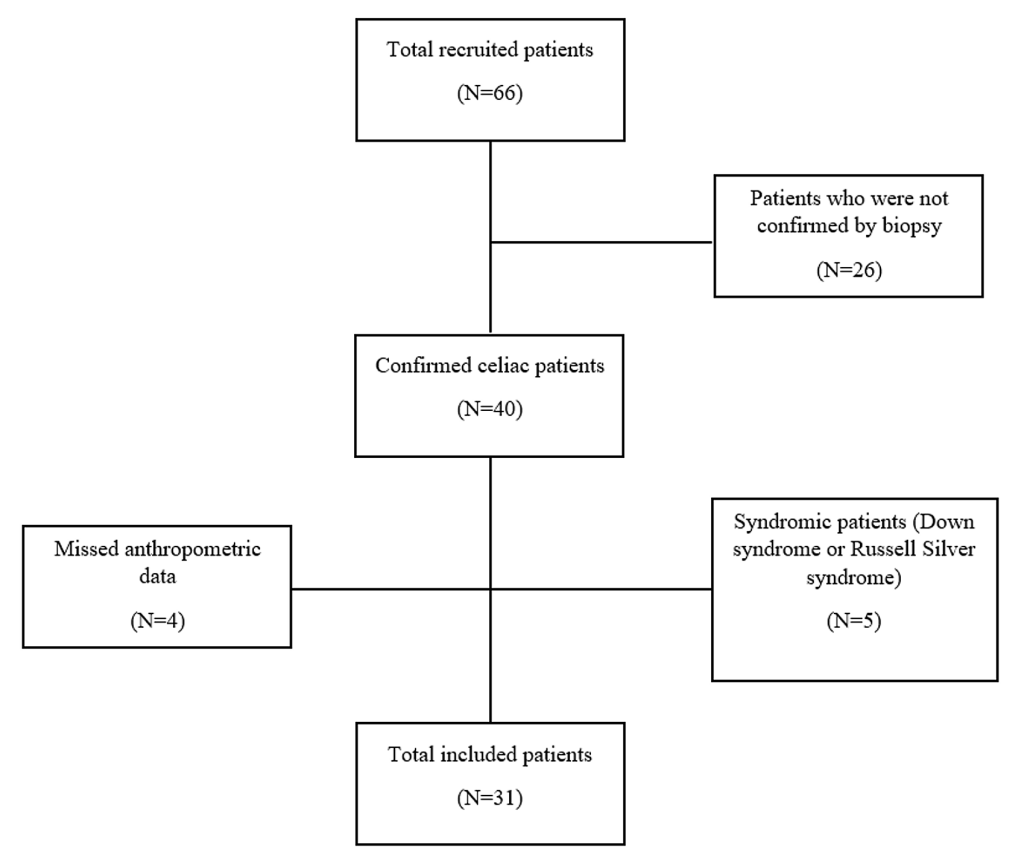

FIGURE 1: Patients selection process flowchart 


\section{Cureus}

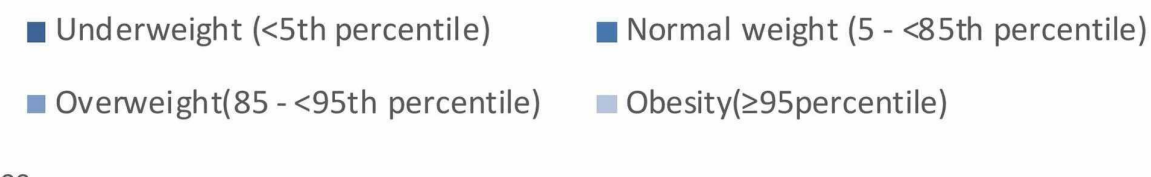

100

90

80

70

60

50

45.2

40

30

20

10

6.5

6.5

0

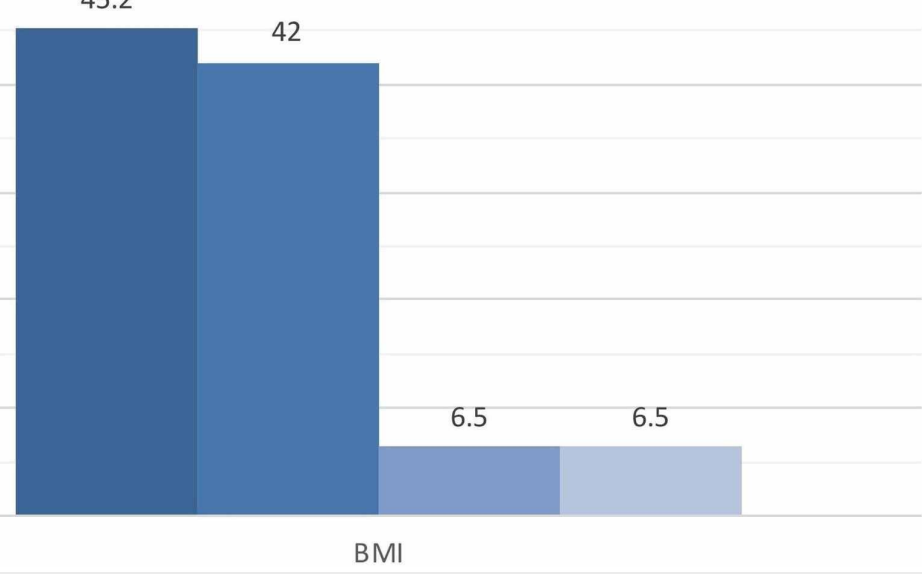

FIGURE 2: Percentages of BMI categories 


\section{Cureus}

- Short stature ( $<5$ th percentile)

- Normal stature(5-<95th percentile)

- Tall stature( $\geq 95$ th percentile)

100

90

80

70

60

58

50

40

35.5

30

20

10

6.5

0

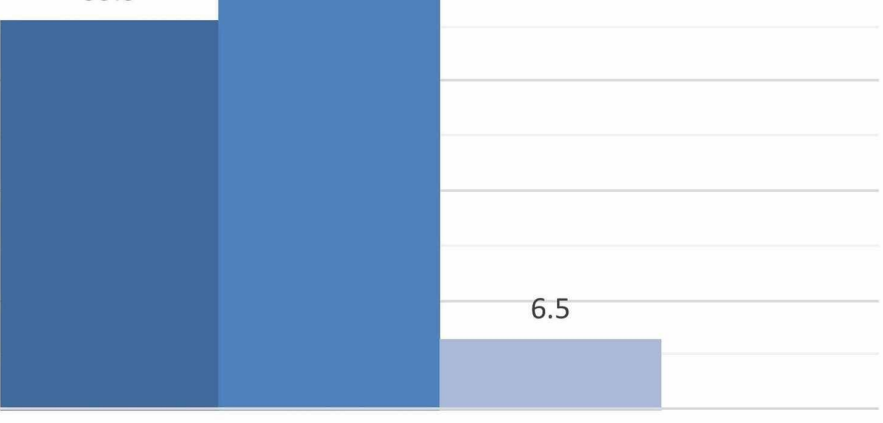

Height

FIGURE 3: Percentages of height categories 


\section{Cureus}

\begin{tabular}{|ll|}
\hline Characteristic & $\mathrm{n}=31$ \\
\hline Mean age $( \pm \mathrm{SD})$ & $7.71( \pm 3.09)$ \\
\hline Gender & Male: $19(61.29 \%)$ \\
\hline Mean weight at diagnosis $( \pm \mathrm{SD})$ & Female: $12(38.70 \%)$ \\
Mean height at diagnosis $( \pm \mathrm{SD})$ & $23.45 \mathrm{Kg}( \pm 12.18)$ \\
Mean BMl at diagnosis $( \pm \mathrm{SD})$ & $119.77 \mathrm{~cm}( \pm 18.27)$ \\
Associated co-morbidities & $15.44( \pm 3.65)$ \\
Diabetes mellitus type I & \\
Asthma & $9(29.0 \%)$ \\
Hereditary anemia & $5(16.1 \%)$ \\
ADHD & $2(6.5 \%)$ \\
Autism & $2(6.5 \%)$ \\
\hline
\end{tabular}

TABLE 1: Patients' demographic and clinical characteristics

ADHD - attention deficit hyperactivity disorder

\begin{tabular}{|c|c|c|c|}
\hline Anthropometric measurements & Percentile & Z-score & P-value (*) \\
\hline Mean weight for age ( \pm SD) & $25.32( \pm 34.79)$ & $-1.37( \pm 1.80)$ & 0.0002 \\
\hline Mean height for age ( \pm SD) & $30.69( \pm 31.38)$ & $-0.92( \pm 1.46)$ & 0.0016 \\
\hline Mean BMI for age ( $\pm S D)$ & $28.74( \pm 34.01)$ & $1.28( \pm 1.93)$ & 0.0004 \\
\hline
\end{tabular}

TABLE 2: Anthropometric measurements of patients at the time of diagnosis

* T-test: compared to the normal population mean z-score (0).

\begin{tabular}{|c|c|c|c|}
\hline Anthropometric measurements & Non-comorbid & Comorbid & P-value \\
\hline Mean weight for age $z$-score $( \pm S D)$ & $-1.50( \pm 1.71)$ & $-1.29( \pm 1.90)$ & 0.62 \\
\hline Mean height for age z-score ( $\pm S D)$ & $-1.00( \pm 1.47)$ & $-0.88( \pm 1.49)$ & 0.73 \\
\hline Mean BMI for age z-score ( \pm SD) & $-1.37( \pm 1.67)$ & $-1.23( \pm 2.13)$ & 0.73 \\
\hline
\end{tabular}

TABLE 3: Mean z-score of anthropometric measurements comparison between non-comorbid and comorbid patients

\section{Discussion}

In this single-center retrospective review study, we reviewed the anthropometric measures of 31 biopsyconfirmed celiac patients to estimate their growth variations. Results showed that $45.16 \%$ of included patients were underweight at the time of diagnosis, $41.9 \%$ were normal weight, $6.5 \%$ were overweight, and $6.5 \%$ were obese. Additionally, $35 \%$ of patients had short stature at the time of diagnosis.

Our findings in terms of weight categories were consistent with the classical belief that CD presents with 
malabsorption. In 2004, an American study conducted by Hoffenberg EJ et al. showed that children with seropositive CD had significantly lower z-scores for weight, height, and BMI compared to other children [11]. More recently, a study conducted in Italy found that the frequency of overweight and obesity at the time of $\mathrm{CD}$ diagnosis was notably reduced compared to the general population. This reduction persisted even during the application of the gluten-free diet [12]. Some studies link this decrease in BMI to other factors such as the extent of the mucosal injury, diarrhea at the time of diagnosis, and female sex [13].

On the contrary, in the United States, Reilly et al. found that the majority of their celiac patients (142) had a normal BMI at the time of diagnosis (74.5\%), and more patients were found to be overweight (19\%) than underweight (6.5\%) [10]. It is important to note that their study had a larger sample size, explaining why our results are different. Locally at King Abdulaziz University, a retrospective study was conducted on 80 celiac pediatric patients to describe the clinical pattern of the disease. The study found that only $23 \%$ of patients were underweight at the time of diagnosis, while the majority of their patients presented with a normal BMI. However, the study also showed that $38 \%$ of their patients had short stature at the time of diagnosis, which is very similar to our research finding (35\%) [14].

Even though our results generally show that our patients are significantly underweight, it is important to note that having a normal or high BMI does not rule out the possibility of celiac disease. This point is reaffirmed in our findings since $54.9 \%$ of our patients had normal or high BMI at the time of diagnosis.

The pathogenesis behind the atypical weight presentation of celiac disease is explained by the "compensatory" hypothesis [5]. The damaged atrophic villi are countered by compensatory mechanisms in the distal, functionally preserved parts of the intestines. An increase in the number of epithelial cells, the height of villi and the depth of the crypt cells compose the morphological adaptations that will increase nutrient absorption level [5]. Hypothetically, when these measures overcompensate the proximal villous damage, it may lead to an excessive caloric extraction and thus leads to an overweight or obese presentation [5]. The presence and severity of CD's classical symptoms are principally affected by the capability and efficiency of the compensatory mechanisms rather than the degree of villous atrophy or the extent of intestinal insult $[15,16]$. However, since these adaptations are time-dependent, they increase as age increases, which may explain the tendency of atypical presentation in older children and adolescents [5].

Regarding the reliability of the presence of malabsorption for clinical suspicion of CD, van der Pals et al. conducted a study with a large sample of 12,632 12-year-old children screened for CD, and their anthropometric measures were recorded at the time of diagnosis. The study concluded that children with CD had lower weight, height and BMI compared to children with no CD, yet at an individual level, their growth parameters were not a reliable input in the diagnosis of $\mathrm{CD}[8]$.

CD and type 1 diabetes share a common genetic and possibly an environmental basis for their pathogenesis [17]. Therefore, the two conditions frequently present together. In fact, it is estimated that the global risk of CD complicating type 1 diabetes ranges from $2.5 \%$ to 16.4 [18]. This supports our results since $29 \%$ of our CD patients had type 1 diabetes. However, our results show no significant differences in the z-scores of any of the anthropometric measures between non-comorbid and comorbid (including Type 1 diabetes) groups. On the contrary, Tsouka et al. study showed that children with CD and T1DM had significantly higher anthropometric measures and a lower incidence of anemia compared to children with CD alone [19]. This might be attributed to the anabolic effect of insulin therapy [20].

Limitations to our research include missing data from patient files due to the retrospective nature of our study. Moreover, our research had a small sample size, which can be attributed partly to many files being missing or lost during the transition of paper files to the new electronic medical record system in King Abdulaziz Medical City. We also used the CDC growth charts, which might not represent our population accurately.

\section{Conclusions}

Based on our findings, we concluded that celiac patients in our center have a significantly lower BMI, weight for age, and height for age at the time of diagnosis. We also conclude that having normal or high growth parameters does not exclude the possibility of celiac disease. Our recommendations for this topic's future research is to increase the sample size by including more patients from other centers in our region. We also recommend measuring the effectiveness of the gluten-free diet on the growth parameters of celiac patients and the progression of the condition. Finally, we suggest using a customized growth chart that is more representative of our local population.

\section{Additional Information \\ Disclosures}

Human subjects: Consent was obtained by all participants in this study. Animal subjects: All authors have confirmed that this study did not involve animal subjects or tissue. Conflicts of interest: In compliance with the ICMJE uniform disclosure form, all authors declare the following: Payment/services info: All 
authors have declared that no financial support was received from any organization for the submitted work. Financial relationships: All authors have declared that they have no financial relationships at present or within the previous three years with any organizations that might have an interest in the submitted work.

Other relationships: All authors have declared that there are no other relationships or activities that could appear to have influenced the submitted work.

\section{References}

1. Saari A, Harju S, Mäkitie O, Saha MT, Dunkel L, Sankilampi U: Systematic growth monitoring for the early detection of celiac disease in children. JAMA Pediatr. 2015, 169:e1525. 10.1001/jamapediatrics.2015.25

2. Gujral N, Freeman HJ, Thomson AB: Celiac disease: prevalence, diagnosis, pathogenesis and treatment . World J Gastroenterol. 2012, 14:6036-6059. 10.3748/wig.v18.i42.6036

3. Safi MA: Prevalence of celiac disease in saudi arabia: meta-analysis . Glob Vaccines Immunol. 2018, 3:1-6. 10.15761/GVI.1000134

4. Kinos S, Kurppa K, Ukkola A, et al.: Burden of illness in screen-detected children with celiac disease and their families. J Pediatr Gastroenterol Nutr. 2012, 55:412-416. 10.1097/MPG.0b013e31825f18ff

5. Diamanti A, Capriati T, Basso M, et al.: Celiac disease and overweight in children: an update . Nutrients. 2014, 6:207-220. 10.3390/nu6010207

6. Rostom A, Murray JA, Kagnoff MF: American Gastroenterological Association (AGA) Institute technical review on the diagnosis and management of celiac disease. Gastroenterology. 2006, 131:1981-2002. 10.1053/j.gastro.2006.10.004

7. Hill ID, Dirks MH, Liptak GS, et al.: Guideline for the diagnosis and treatment of celiac disease in children: recommendations of the North American Society for Pediatric Gastroenterology, Hepatology and Nutrition. J Pediatr Gastroenterol Nutr. 2005, 40:1-19.

8. van der Pals M, Myléus A, Norström F, et al.: Body mass index is not a reliable tool in predicting celiac disease in children. BMC Pediatr. 2014, 14:165.

9. Saeed A, Assiri A, Assiri H, Ullah A, Rashid M: Celiac disease in Saudi children: evaluation of clinical features and diagnosis. Saudi Med J. 2017, 38:895-899. 10.15537/smj.2017.9.20808

10. Reilly NR, Aguilar K, Hassid BG, et al.: Celiac disease in normal-weight and overweight children: clinical features and growth outcomes following a gluten-free diet. J Pediatr Gastroenterol Nutr. 2011, 53:528-531.

11. Hoffenberg EJ, Emery LM, Barriga KJ, et al.: Clinical features of children with screening-identified evidence of celiac disease. Pediatrics. 2004, 113:1254-1259. 10.1542/peds.113.5.1254

12. Brambilla P, Picca M, Dilillo D, et al.: Changes of body mass index in celiac children on a gluten-free diet . Nutr Metab Cardiovasc Dis. 2013, 23:177-182. 10.1016/j.numecd.2011.10.002

13. Cheng I, Brar PS, Lee AR, Green PH: Body mass index in celiac disease: beneficial effect of a gluten-free diet J Clin Gastroenterol. 2010, 44:267-271. 10.1097/MCG.0b013e3181b7ed58

14. Saadah OI: Celiac disease in children and adolescents at a singe center in Saudi Arabia . Ann Saudi Med. 2011, 31:51-57. 10.4103/0256-4947.75779

15. Brar P, Kwon GY, Egbuna II, Holleran S, Ramakrishnan R, Bhagat G, Green PH: Lack of correlation of degree of villous atrophy with severity of clinical presentation of coeliac disease. Dig Liver Dis. 2007, 39:26-29. 10.1016/j.dld.2006.07.014

16. Murray JA, Rubio-tapia A, Van Dyke CT, et al.: Mucosal atrophy in celiac disease: extent of involvement, correlation with clinical presentation, and response to treatment. Clin Gastroenterol Hepatol. 2008, 6:186193. 10.1016/..cgh.2007.10.012

17. Hagopian W, Lee HS, Liu E, et al.: Co-occurrence of type 1 diabetes and celiac disease autoimmunity . Pediatrics. 2017, 140:e20171305. 10.1542/peds.2017-1305

18. Szaflarska-Popławska A: Coexistence of coeliac disease and type 1 diabetes . Prz Gastroenterol. 2014, 9:1117. 10.5114/pg.2014.40844

19. Tsouka A, Mahmud FH, Marcon MA: Celiac disease alone and associated with type 1 diabetes mellitus . J Pediatr Gastroenterol Nutr. 2015, 61:297-302. 10.1097/MPG.0000000000000789

20. De Vries L, Bar-Niv M, Lebenthal Y, et al.: Changes in weight and BMI following the diagnosis of type 1 diabetes in children and adolescents. Acta Diabetol. 2014, 51:395-402. 10.1007/s00592-013-0524-4 